ROCZNIKI TEOLOGICZNE

Tom LXVIII, zeszyt $3-2021$

DOI: https://doi.org/10.18290/rt.21683-2

\title{
DIE ANTWORT DER KATHOLISCHEN KIRCHE AUF DIE FRAGE DER PERSONEN MIT LEBENSVERKÜRZENDEN ERKRANKUNGEN IM LICHT DES SCHREIBENS DER GLAUBENSKONGREGATION SAMARITANUS BONUS UND IM KONTEXT DES URTEILS DES POLNISCHEN VERFASSUNGSGERICHTS VOM 22.10.2020
}

\author{
THE RESPONSE OF THE CATHOLIC CHURCH TO THE ISSUE OF PERSONS \\ WITH PATHOLOGIES "INCOMPATIBLE WITH LIFE" IN THE LIGHT \\ OF THE DOCUMENT OF THE CONGREGATION FOR THE FAITH SAMARITANUS BONUS \\ AND IN CONTEXT OF THE DECISION OF THE POLISH CONSTITUTIONAL COURT \\ OF OCTOBER 22, 2020
}

\begin{abstract}
A b s t r a c t. The decision of the Polish Constitutional Court of 22.10.2020 regarding the non-conformity of the embryopathic indication for abortion with the constitution has triggered severe protests. At the center of the controversy is the handling of pathologies "incompatible with life", which for many represent an unacceptable evil and a reason for abortion. In contrast, the Church has always stood on the ground of unconditional respect for the life of every human person. In the article, starting from the court decision the question of persons with pathologies "incompatible with life" is examined ethically in the light of a new document of the Congregation for the Doctrine of the Faith, Samaritanus bonus, as well as in the broader perspective of the magisterial teaching to date.
\end{abstract}

Keywords: "incompatible with life"; abortion; Samaritanus bonus; Polish Constitutional Court; person.

Dr. theol. ANDRZEJ KUCIŃSKI - in Moraltheologie an der Universität Bonn promoviert, Priester des Erzbistums Köln, Mitarbeiter der Glaubenskongregation, wohnhaft in Rom; e-mail: a.kucinski@live.com; ORCID: https//orcid.org/0000-0001-5327-1670.

Ks. dr ANDRZEJ KUCIŃSKI - doktor teologii moralnej na Uniwersytecie w Bonn, ksiądz archidiecezji kolońskiej, pracownik Kongregacji Nauki Wiary, mieszka w Rzymie; e-mail: a.ku cinski@live.com; ORCID: https//orcid.org/0000-0001-5327-1670. 


\section{EINLEITUNG}

Mit der folgenden Aussage hat das päpstliche Lehramt eine absolute Grenze gesetzt, insofern es in der Autorität Christi einen ganz bestimmten Umgang mit beginnendem menschlichen Leben einfordert:

Mit der Autorität, die Christus Petrus und seinen Nachfolgern übertragen hat, erkläre ich deshalb in Gemeinschaft mit den Bischöfen - die mehrfach die Abtreibung verurteilt und, obwohl sie über die Welt verstreut sind, bei der eingangs erwähnten Konsultation dieser Lehre einhellig zugestimmt haben - daß die direkte, das heißt als Ziel oder Mittel gewollte Abtreibung immer ein schweres sittliches Vergehen darstellt, nämlich die vorsätzliche Tötung eines unschuldigen Menschen ${ }^{1}$.

Damit sind die Bedingungen einer unfehlbaren Lehre erfüllt, da es um den sog. „Zweiten“ Bereich der Lehrverkündigung geht, zu dem die Wahrheiten aus dem Bereich Glaube oder Sitten gehören, welche in einem notwendigen Zusammenhang mit der Offenbarung stehen und daher einen unwiderruflichen Charakter haben ${ }^{2}$. Daraus ergibt sich, dass gemäß der katholischen Lehre jeder Gläubige daran gehalten ist, der Feststellung, dass es sich bei Abtreibung um eine vorsätzliche Tötung eines Unschuldigen handelt, seine feste und endgültige Zustimmung zu geben, andernfalls ,lehnt [er] Wahrheiten der katholischen Kirche ab und steht deshalb nicht mehr in der vollen Gemeinschaft mit der katholischen Kirche“3. Der 2014 heiliggesprochene Papst Johannes Paul II. erklärt in seiner Enzyklika auch die Konsequenzen der obigen Lehräußerung, indem er unter Berufung auf das Naturrecht und das Gesetz

\footnotetext{
${ }^{1}$ Johannes Paul II., Enzyklika „Evangelium vitae“ über den Wert und die Unantastbarkeit des menschlichen Lebens (25. März 1995), Nr. 62, AAS 87(1995): 477, Zugriff am 16.12. 2020, https://www.vatican.va/content/john-paul-ii/de/encyclicals/documents/hf_jp-ii_enc_25031995_ evangelium-vitae.html.

${ }^{2} \mathrm{Vgl}$. CIC c. 750 § 2: „Fest anzuerkennen und zu halten ist auch alles und jedes, was vom Lehramt der Kirche bezüglich des Glaubens und der Sitten endgültig vorgelegt wird, das also, was zur unversehrten Bewahrung und zur getreuen Darlegung des Glaubensgutes erforderlich ist; daher widersetzt sich der Lehre der katholischen Kirche, wer diese als endgültig zu haltenden Sätze ablehnt“. - Ergänzung des CIC 1983 nach dem Ap. Schreiben „Ad tuendam fidem “, durch das einige Normen in den CIC und in den CCEO eingefügt werden (18. Mai 1998), Nr. 4, AAS 90(1998): 459-60, Zugriff am 16.12.2020, https://www.vatican.va/roman_cu ria/congregations/cfaith/documents/rc_con_cfaith_doc_1998_professio-fidei_ge.html; vgl. auch Kongregation für die Glaubenslehre, Lehrmäßiger Kommentar zur Schlussformel der „Professio fidei“ (29. Juni 1998), Nr. 7-9, AAS 90(1998): 547-8 (Beigabe im Internet - ebd.).

${ }^{3}$ Kongregation für die Glaubenslehre, Lehrmäßiger Kommentar, Nr. 6, AAS 90 (1998): 547.
} 
Gottes auf die unbedingte und dauerhafte Unerlaubtheit einer solchen Handlung nicht zuletzt gegenüber anderslautenden positiven Gesetzen verweist:

Kein Umstand, kein Zweck, kein Gesetz wird jemals eine Handlung für die Welt statthaft machen können, die in sich unerlaubt ist, weil sie dem Gesetz Gottes widerspricht, das jedem Menschen ins Herz geschrieben, mit Hilfe der Vernunft selbst erkennbar und von der Kirche verkündet worden ist ${ }^{4}$.

Kurzum: Von der sittlichen Missbilligung der Abtreibung gibt es nach der geltenden Lehre der Kirche keine Ausnahmen.

Nun aber sprach das polnische Verfassungsgericht am 22.10.2020, ausgerechnet dem Gedenktag des Heiligen Johannes Paul II., sein Urteil aus, gemäß dem die bisher geltende, embryopathische Indikation für die straffreie Abtreibung mit der Verfassung unvereinbar sei und somit der Gesetzgeber eine Anpassung des entsprechenden Gesetzes vornehmen solle:

Das Gericht $[\ldots]$ stellte [...] fest, dass die Legalisierung des Abtreibungsverfahrens, wenn pränatale Untersuchungen oder andere medizinische Indikationen auf die große Wahrscheinlichkeit einer schweren und irreversiblen Behinderung des Fötus oder einer unheilbaren lebensbedrohlichen Erkrankung hinweisen, keine verfassungsrechtliche Rechtfertigung besitzt ${ }^{5}$.

Dieses Urteil führte zu Massenprotesten in der polnischen Bevölkerung, verbunden mit einer nie dagewesenen Gewalttätigkeit. Insbesondere die massive Beteiligung von jungen Menschen und die Vehemenz der Kritik an der katholischen Kirche, die bis zur Störung von Gottesdiensten, tätlichen Angriffen auf Priester und Verunreinigung von kirchlichen Gebäuden reichte, war für viele überraschend und erschreckend. In zahlreichen Medien entflammten daraufhin heftige Debatten, viele Familien wurden gespalten. Alles in allem erschien die polnische Nation in der Weltöffentlichkeit als tiefzerrissene Gesellschaft, in der die meisten wohl die Möglichkeit der Abtreibung von

\footnotetext{
${ }^{4}$ Johannes Paul II., Enzyklika „Evangelium vitae“, Nr. 62, AAS 87(1995): 472.

5 „Trybunał [...] stwierdził [...] że legalizacja zabiegu przerwania ciąży, w przypadku gdy badania prenatalne lub inne przesłanki medyczne wskazują na duże prawdopodobieństwo ciężkiego i nieodwracalnego upośledzenia płodu albo nieuleczalnej choroby zagrażającej jego życiu, nie znajduje konstytucyjnego uzasadnienia“. - „Komunikat po ogłoszeniu wyroku w sprawie ,Planowanie rodziny, ochrona płodu ludzkiego i warunki dopuszczalności przerywania ciąży““, Trybunał Konstytucyjny, Zugriff am 16.12.2020, https://trybunal.gov.pl/ postepowa nie-i-orzeczenia/komunikaty-prasowe/komunikaty-po/art/11299-planowanie-rodziny-ochrona-plo du-lu dzkiego-i-warunki-dopuszczalnosci-przerywania-ciazy (alle Übersetzungen vom Verf.).
} 
schwer behinderten Föten, wenn nicht als Recht der Mutter, dann zumindest als „kleineres“, „notwendiges“ Übel ansehen. Hierbei muss zu bedenken gegeben werden, dass es sich um eine Bevölkerung handelt, die zum übergroßen Teil der katholischen Kirche angehört und in der bisher der Katholizismus als allen Krisen der westlichen Gesellschaften trotzende, identitätskonstituierende Kraft galt.

Fast genau einen Monat vor dem Urteil des Verfassungsgerichts, am 26. September, wurde in Rom das neue Dokument der Glaubenskongregation Samaritanus bonus vorgestellt ${ }^{6}$. In einem Unterkapitel dieses Schreibens (V.6) werden ausgerechnet die Begleitung und die Fürsorge in vorgeburtlichem und pädiatrischem Alter angeschnitten, womit u. a. das Thema von den sog. lebensverkürzenden Erkrankungen und Abtreibung bei embryopathischer Indikation zur Sprache kommt.

Die immer noch andauernde gesellschaftliche Auseinandersetzung um das Gerichtsurteil in Polen zeigt jedoch deutlich, dass das Bewusstsein der Katholiken, welche moralische Verpflichtungen sie betreffen, vor allem bzgl. deren Begründung weiterhin schwindet. Insofern bietet das jüngste Schreiben der Glaubenskongregation eine Gelegenheit, der erwähnten kirchlichen Position nachzugehen und die lehramtliche Argumentation zugunsten des „Lebenlassens“ von fehlgebildeten menschlichen Lebewesen zu vertiefen. Dazu dient in einem ersten Schritt ein grober Überblick über den Umgang mit lebensverkürzenden Erkrankungen im Hinblick auf die entsprechende Abtreibungsindikation, wobei hier keine detaillierte Darstellung der medizinischen Aspekte erfolgen kann. Im zweiten Schritt sollen die einschlägigen Passagen von Samaritanus bonus präsentiert werden, im dritten erfolgt die Kontextualisierung durch den Rekurs auf ausgewählte Dokumente des universalkirchlichen Lehramtes. Im Ergebnis soll sich die Entfaltung und die Kontinuität der kirchlichen Lehre in diesem Thema zeigen, in die sich nun auch Samaritanus bonus einfügt.

\section{DIE „LEBENSVERKÜRZENDEN ERKRANKUNGEN““ IM ÜBERBLICK}

„Als ,lebensverkürzende Erkrankungen' werden nach dem heutigen Stand der Medizin unheilbare, progredient verlaufende und zum Tod in der Kindheit

\footnotetext{
${ }^{6}$ Kongregation für die Glaubenslehre, „Samaritanus bonus“. Schreiben über die Sorge an Personen in kritischen Phasen und in der Endphase des Lebens (14. Juli 2020) (Libreria Editrice Vaticana: Città del Vaticano, 2020), Zugriff am 16.12.2020, https://www.vatican.va/roman _curia/congregations/cfaith/documents/rc_con_cfaith_doc_20200714_samaritanus-bonus_ge.html.
} 
oder Jugend führende Erkrankungen bezeichnet, wie z. B. neuromuskuläre und genetische Krankheiten sowie schwere Stoffwechselstörungen"7, heißt es in einer deutschen Definition. Eine polnische Entsprechung berücksichtigt dagegen unterschiedliche Todeszeitpunkte der erkrankten Kinder einschließlich der Zeit vor der Geburt. Demnach könne eine lebensverkürzende Krankheit zu 1) einer Fehlgeburt, 2) der vorzeitigen Geburt eines kranken Kindes, 3) dem Tod des Kindes unmittelbar nach der Geburt oder in einem frühen Lebensstadium führen, und zwar trotz jeglicher Behandlungsversuche ${ }^{8}$. Solche lebensverkürzenden Krankheiten (wady letalne) sind ein besonderer Fall der im polnischen Gesetz zum Schutz des ungeborenen Lebens vorkommenden embryopathologischen Indikation, die durch das Verfassungsgericht vor kurzem in Frage gestellt wurde.

Die Zahl der jährlich in Polen geborenen Kinder mit einer lebensverkürzenden Erkrankung wird in der Literatur auf über 600 geschätzt ${ }^{9}$. Je älter die Mutter, desto höher das Risiko einer solchen Erkrankung. Mittels der Pränataldiagnostik lassen sich manche Erkrankungen dieser Art bereits in einem frühen Schwangerschaftsstadium erkennen ${ }^{10}$. Das polnische Gesetz erlaubt bisher in dem Fall die Abtreibung ${ }^{11}$. Wird das Kind jedoch lebend geboren, stehen die Eltern vor einer Reihe von schwerwiegenden Entscheidun-

\footnotetext{
${ }^{7}$ Andrea Pyttlik und Johannes Jungbauer, „Im Leben, im Sterben und darüber hinaus Eine qualitative Studie zur Trauerbegleitung von Eltern lebensverkürzend erkrankter Kinder“, in Wegbegleitung, Trost und Hoffnung. Interdisziplinäre Beiträge zum Umgang mit Sterben, Tod und Trauer, hrsg. v. Johannes Jungbauer, Rainer Krockauer (Opladen u. a., 2013), 125.

8 Vgl. Aneta Jarzębińska, „Od diagnozy do towarzyszenia w żałobie - wsparcie rodzin z dzieckim z wadą letalna““, Interdyscyplinarne Konteksty Pedagogiki Specjalnej 22 (2018): 377, Zugriff am 16.12.2020, https://pressto.amu.edu.pl/index.php/ikps/article/view/21199/20455; vgl. auch: Tomasz Dangel, „Wady letalne u noworodków. Opieka paliatywna jako alternatywa wobec aborcji i uporczywej terapii“, Standardy Medyczne 31, Nr. 9(2007): 108-10, Zugriff am 16.12. 2020, https://hospicjum.waw.pl/pliki/Artykul/1095_wadyletalneunoworodkow-2007. pdf.

${ }^{9}$ Vgl. Jarzębińska, „Od diagnozy“, 377.

${ }^{10} \mathrm{Vgl}$. ebd.; vgl. auch Jana Skrzypczak und Jakub Kornacki, „Ciąża o przebiegu niepowikłanym“, in Położnictwo. Podręcznik dla połoznych i pielęgniarek, hrsg. v. Grzegorz H. Bębrowicz (Warszawa: PZWL, 2005), 72-4.

11 Das polnische Gesetz „über Familienplanung, Schutz des menschlichen Fötus und Zulassungsbedingungen für Abtreibung“ vom 7.1.1993 sieht drei Fälle der Straffreiheit von Abtreibung vor: seriöser Verdacht auf schwere, irreversible Fehlbildung oder eine unheilbare Erkrankung des Fötus, Gefährdung der Gesundheit oder des Lebens der Mutter sowie Schwangerschaftsverursachung durch eine Straftat - vgl. „Ustawa z dnia 7 stycznia 1993 r. o planowaniu rodziny, ochronie płodu ludzkiego i warunkach dopuszczalności przerywania ciąży“, Internetowy System Aktów Prawnych, Zugriff am 01.02.2021, https://isap.sejm.gov.pl/isap.nsf/ download.xsp/WDU19930170078/U/D19930078Lj.pdf.
} 
gen (je nach Art und Grad der Erkrankung): Reanimation oder nicht; Intensivtherapie im Krankenhaus oder Palliativbehandlung zu Hause, Verbleib im Krankenhaus oder in einer besonderen Einrichtung; Versuch einer chirurgischen Behandlung der Erkrankung oder deren Unterlassung; Tod des Kindes im Krankenhaus oder zu Hause ${ }^{12}$. Für eine moralische Orientierung ist die Erkenntnis von Belang, dass das Vorkommen von unheilbaren pathologischen Entwicklungen in der pränatalen bzw. postnatalen Phase als natürliche, d. h. der menschlichen Unvollkommenheit entsprechende Phänomene zu gelten haben ${ }^{13}$. Erkrankungen solcher Art machen jedoch in Polen in der Praxis keinesfalls den größten Anteil der Abtreibungen aufgrund der besagten Indikation aus (dieser Platz „gebührt“ der Trisomie 21 mit durchschnittlich 37\% aller Schwangerschaftsabbrüche $)^{14}$. Trotzdem, wie in den letzten Protesten deutlich wurde, dienen sie (bzw. äußerst seltene, aber durch erschreckende Bilder besonders öffentlichkeitswirksame Einzelfälle dieser Art ${ }^{15}$ als Hauptargument gegen eine Verschärfung der diesbezüglichen Gesetzgebung. Laut einer rezenten Studie hatte Polen 2018 jedenfalls die niedrigste legale Abtreibungsrate europaweit ( 3 pro 1000 lebend Geborene) ${ }^{16}$. Es ist zu vermuten, dass es auch die niedrigste Zahl von embryopathisch bedingten Abtreibungen aufweist ${ }^{17}$. Für die drei letzten Jahre sieht die polnische Abtreibungsstatistik wie folgt aus ${ }^{18}$ :

12 Vgl. Jarzębińska, „Od diagnozy“, 378.

${ }^{13}$ Vgl. Tomasz Dangel, „Wady letalne u płodów i noworodków. Opieka paliatywna jako alternatywa wobec eugenicznej aborcji, eugenicznego dzieciobójstwa i uporczywej terapii“, 9, Zugriff am 16.12.2020, https://www.hospicjum.waw.pl/pliki/Artykul/1132_wadyletalneuplodo winoworodkow.pdf.

${ }^{14}$ Filip Furman, ,Jakie skutki wywołuje wyrok Trybunału Konstytucyjnego w sprawie warunków dopuszczalności aborcji?“, 2, Zugriff am 21.12.2020, https://ordoiuris.pl/sites/default/ files/inline-files/Stanowisko_CNSiB_1.pdf.

$15 \mathrm{Vgl}$. ebd.

${ }^{16} \mathrm{Vgl}$. „Number of abortion procedures performed in European countries in 2018“, Statista, accessed December 16, 2020, https://www.statista.com/statistics/866423/abortion-rateeurope/.

${ }^{17}$ Nach den Daten von 2005 aus dem Artikel: Dangel, „Wady letalne u płodów i noworodków“, 2.

${ }^{18}$ Vgl. Furman, ,Jakie skutki“, 4-5. 


\begin{tabular}{|c|c|c|c|c|}
\hline $\begin{array}{l}\text { Legale Abtreibungen nach einzelnen Indikatio- } \\
\text { nen }\end{array}$ & $\begin{array}{l}\text { Mittelwert } \\
\text { 2009-2019 }\end{array}$ & 2019 & 2018 & 2017 \\
\hline Insgesamt & 881,45 & 1110 & 1076 & 1057 \\
\hline $\begin{array}{l}\text { Gefährdung für Gesundheit oder Leben der } \\
\text { Mutter }\end{array}$ & 36,55 & 33 & 25 & 22 \\
\hline $\begin{array}{l}\text { Verdacht auf kriminelle Schwangerschaftsur- } \\
\text { sache }\end{array}$ & 1,18 & 3 & 1 & 0 \\
\hline $\begin{array}{l}\text { Seriöser Verdacht auf schwere, irreversible } \\
\text { Fehlbildung oder eine unheilbare Erkrankung } \\
\text { des Fötus }\end{array}$ & 843,73 & 1074 & 1050 & 1035 \\
\hline $\begin{array}{l}\text { Prozentanteil von Abtreibungen bei embryo- } \\
\text { pathologischer Indikation an allen legalen } \\
\text { Schwangerschaftsabbrüchen }\end{array}$ & $95,52 \%$ & $96,76 \%$ & $97,58 \%$ & $97,92 \%$ \\
\hline
\end{tabular}

Daraus lässt sich schließen, dass Abtreibungen mit embryopathologischer Indikation fast die Gesamtzahl der legalen Schwangerschaftsabbrüche in Polen ausmachen. Abgesehen von der Tatsache, dass nur ein Teil davon tatsächlich Fälle betreffen, in denen lebensverkürzende Erkrankungen festgestellt werden (neben anderen, die nicht zum perinatalen Tod führen), zeigt dieser Befund - nicht zuletzt vor dem Hintergrund der jüngsten Proteste und der sich hieraus ableitenden Vermutung eines fortschreitenden Verlustes der ethischen Glaubenskompetenz vieler polnischer Katholiken -, dass eine argumentative Begründung der geltenden Lehre der Kirche zur Abtreibung, spezifiziert besonders im Hinblick auf lebensverkürzende Erkrankungen nottut. Dafür bietet Samaritanus bonus einen aktuellen und guten Anknüpfungspunkt.

\section{SAMARITANUS BONUS UND DIE „KLEINEN PATIENTEN“}

Selbstredend kann man die Behandlung von Personen mit lebensverkürzenden Erkrankungen nur innerhalb der Gesamtverkündigung des universalkirchlichen Lehramts zur Abtreibung betrachten. Doch widmet Samaritanus bonus ausgerechnet diesem Aspekt der Problematik ein ganzes Unterkapitel in Teil V (19-49) ${ }^{19}$, in welchem es um einzelne moralische, medizinische, pastorale, rechtliche und bildungsethische Probleme geht, die mit der Sorge an Personen in kritischen Lebensstadien zu tun haben. Diesem längsten Teil gehen, neben der Einführung (3-5), folgende Passagen voraus: (I) ein Einleitungskapitel mit einigen Grund-

\footnotetext{
${ }^{19}$ In diesem Abschnitt werden in Klammern jeweils die Seiten der deutschen Ausgabe des Schreibens angegeben.
} 
sätzen der Fürsorge, welche den Patienten ins Zentrum stellen (5-9); (II) eine biblisch-theologische Untermauerung durch Rekurs auf das Geheimnis des leidenden Christus am Kreuz mit den unter diesem Kreuz stehenden Personen (9-13); (III) die Betonung der Unantastbarkeit, der Heiligkeit und der Positivität menschlichen Lebens (13-16) und (IV) die Analyse der Faktoren zuungunsten der Anerkennung dieses Fundamentalwertes (16-19).

Das Unterkapitel V.6 Die Begleitung und die Fürsorge in vorgeburtlichem und pädiatrischen Alter behandelt das Thema der Kinder mit schweren perinatalen Erkrankungen ausgehend vom folgenden Grundsatz:

Seit der Zeugung sind Kinder, die an Missbildungen oder Erkrankungen jeglicher Art leiden, kleine Patienten, wobei die Medizin heutzutage immer in der Lage ist, ihnen beizustehen und sie auf eine gegenüber dem Leben respektvolle Weise zu begleiten. Ihr Leben ist heilig, einzigartig, unwiederholbar und unantastbar, genau wie das jeder erwachsenen Person (34, Hervorhebung im Original).

Damit werden diese menschlichen Lebewesen anthropologisch und ethisch den erwachsenen Personen gleichgestellt, womit ein unbedingter Anspruch auf die Achtung ihrer Menschenwürde und damit auf entsprechende - laut dem Schreiben immer mögliche - medizinische Behandlung trotz keinerlei Aussicht auf Genesung verbunden sei: Sie ,dürfen [...] in keiner Weise aus der Fürsorge ausgeschlossen werden, sondern sie müssen bis zum Eintritt des natürlichen Todes wie jeder andere Patient begleitet werden“ (34). Ferner lenkt die Glaubenskongregation die Aufmerksamkeit auf die perinatale Palliativversorgung, die vor allem mittels perinataler Hospize „eine wesentliche Unterstützung " für die Familien bilde (34). In diesem Zusammenhang kommt es nun zu einer negativen Beurteilung des Gebrauchs von Pränataldiagnostik mit ,eugenische[r] Mentalität“, ein Phänomen, das zu einer kulturell bedingten Behinderungsfeindlichkeit führe, so dass die dann häufig vorkommende Abtreibung als „Präventionsmaßnahme“ dargestellt werde, während sie als „absichtliche Tötung“ eines Unschuldigen niemals erlaubt sei (35). Bezeichnend ist dabei die in einem relativ kurzen Textabschnitt zweimalige Verwendung des stark wertenden ,in schwerwiegender Weise unzulässig“ (im Orig. gravemente illecito), einmal in Bezug auf die Pränataldiagnostik zu selektiven Zwecken und ein zweites Mal hinsichtlich der Fürsorgeunterlassung bei einem schwerkranken Neugeborenen (35). Des Weiteren zeigt sich, dass die Kongregation in ihrer Aufforderung zur unbedingten Fürsorge offenbar die Strategie der Meidung von Extremen verfolgt: Weder sollten unverhältnismäßige Behandlungsweisen (,therapeutischer Übereifer") angewandt noch der Tod vor- 
weggenommen werden (35-36). Ferner konzentriert sich das Dokument auf Formen der Palliativmedizin bei lebensverkürzenden Erkrankungen unter besonderer Berücksichtigung der zentralen Rolle der Eltern: „Die Aufrechterhaltung der affektiven Bindung zwischen Eltern und Kind ist ein integraler Bestandteil des Pflegeprozesses. Die Beziehung der Versorgung und Begleitung des Kindes durch die Eltern muss mit allen erforderlichen Instrumenten gefördert werden“ (36). Der euthanasieoffenen Verwendung des Konzepts vom „Vorteil des Minderjährigen“, welches für ein utilitaristisches „KostenNutzen-Kalkül“" verwendet werden könnte, erteilt Samaritanus bonus eine klare Absage (36-37). Ziel sei demgegenüber schließlich, „,das Kind bis zu einem würdigen natürlichen Tod zu begleiten“ (37).

Die dargestellten zentralen Gedanken von Samaritanus bonus des Unterkapitels V.6 stellen einiges klar: Nach diesem Dokument habe man bei unheilbar kranken Neugeborenen mit Personen zu tun, deren unantastbare und unverminderte Menschenwürde ihre absichtliche Tötung von vornherein ausschließe. Stattdessen verdienten sie alle Zuwendung und sämtliche konkrete Behandlungsmaßnahmen, die jedem Menschen angemessen seien, was sowohl den Verzicht auf den „therapeutischen Übereifer“, d. h. auf alle therapeutischen Handlungen, die dem Kind im Sterbeprozess nicht (mehr) zugutekommen oder für es übermäßig belastend sind, als auch das Verbot der Fürsorgeunterlassung im Hinblick auf die notwendige Unterstützung der wesentlichen Lebensfunktion des Patienten beinhalte. Als Besonderheit des Dokumentes ist der deutliche Fokus auf die Umgebung des Kindes, vor allem auf die Eltern, welche sowohl Hauptakteure in Begleitung des Sterbeprozesses als auch Zielpersonen des umfassenden Fürsorgekonzeptes in kritischen Lebensstadien seien, zu bezeichnen, eine Auffassung, die im gesamten römischen Schreiben als Leitgedanke fungiert.

\section{DIE KONSEQUENTE VERKÜNDIGUNG DES LEHRAMTES IM HINBLICK AUF DIE ABTREIBUNG}

Die dargestellte Stellungnahme soll nun überblickartig und exemplarisch in Verhältnis zur sonstigen lehramtlichen Verkündigung gesetzt werden. Schon ein oberflächlicher Blick ergibt eine Fülle von kirchlichen Äußerungen zur Abtreibung, in der Gegenwart vor allem seit der Enzyklika Casti connubii von Pius XI. (31.12.1930). Darin expliziert der Papst im Kontext von Schwangerschaftskonflikten mit Gefahr für Gesundheit und Leben der Mutter die Unmöglichkeit, die direkte Tötung eines Unschuldigen zu rechtfertigen, so- 
wohl der Mutter als auch des Kindes ${ }^{20}$. Gleichzeitig verurteilt die Enzyklika auch die Eugenik in Form der Beeinträchtigung einer Eheschließung von Paaren, bei denen genetisch mit behinderter Nachkommenschaft zu rechnen ist.

Auf ausnahmslose Rechtfertigungsunmöglichkeit einer Abtreibung qua Handlung, die als Ziel oder Mittel die Vernichtung eines unschuldigen Lebens verfolgt, verweist mit scharfen Worten Pius XII. in einer Ansprache an die italienischen katholischen Hebammen (1951), und verurteilt dabei sowohl die Abtreibung zum Schutz des Lebens der Mutter als auch die aufgrund des angeblich mangelnden „Lebenswertes“ des Fötus ${ }^{21}$. In einer weiteren Ansprache wendet sich der Papst gegen die Güterabwägung im Hinblick auf die Abtreibung, wiederum mit dem Hinweis auf die Unantastbarkeit des unschuldigen Lebens und auf die Unmöglichkeit der Bestimmung, wessen Leben „wertvoller“ $\operatorname{sei}^{22}$.

Einen wichtigen Meilenstein in dieser Verkündigungslinie bildet die Erklärung der Kongregation für die Glaubenslehre Quaestio de abortu von $1974^{23}$. Nach der Feststellung, dass der Mensch nicht als bloßer Zweck be-

20 „Der ,sozialen und eugenischen Indikation“ sodann kann und soll mit erlaubten, sittlich einwandfreien Mitteln und innerhalb der rechten Grenzen Rechnung getragen werden; aber den Notständen, auf denen diese Indikationen aufbauen, durch Tötung Unschuldiger abhelfen zu wollen, ist töricht und dem Gebot Gottes zuwider, das der Apostel in die Worte kleidet: ,Man darf nicht Böses tun, um damit Gutes zu stiften““ - Pius XI., Enzyklika „Casti connubii“ über die christliche Ehe im Hinblick auf die gegenwärtigen Lebensbedingungen und Bedürfnisse von Familie und Gesellschaft und auf die diesbezüglich bestehenden Irrtümer und Mißbräuche (31. 12.1930), 3a, AAS 22(1930): 563-4 (lat.), Zugriff am 16.12.2020, https://www.stjosef.at/doku mente/casti_connubii.htm (dt.).

21 „Daher gibt es keinen Menschen, keine menschliche Autorität, keine Wissenschaft, keine medizinische, eugenische, soziale, wirtschaftliche, moralische ,Indikation', die einen gültigen Rechtstitel für eine direkte, absichtliche Verfügung über ein unschuldiges menschliches Leben ausstellen oder geben könnte, das heißt, eine Verfügung, die auf seine Zerstörung, entweder als Zweck oder als Mittel für einen anderen Zweck, abzielt, und zwar vielleicht an sich in keiner Weise illegal. So ist zum Beispiel die Rettung des Lebens der Mutter ein sehr edles Ziel; aber die direkte Tötung des Kindes als Mittel zu diesem Zweck ist nicht zulässig. Die direkte Zerstörung des sogenannten, wertlosen Lebens', geboren oder noch nicht geboren, welche vor einigen Jahren in großer Zahl praktiziert wurde, kann in keiner Weise gerechtfertigt werden“. - Pius XII., Discorso alle partecipanti al Congresso della Unione Cattolica Italiana Ostetriche (29. Oktober 1951), AAS 43(1951): 838.

22 „Die Unantastbarkeit des Lebens eines Unschuldigen hängt nicht von seinem höheren oder niedrigeren Wert“ ab - Pius XII., Discorso ai partecipanti al Convegno del „Fronte della Famiglia" e della Federazione delle Associazioni delle Famiglie (27.11.1951), AAS 43(1951): 859.

${ }^{23}$ Heilige Kongregation für die Glaubenslehre, Erklärung über den Schwangerschaftsabbruch „Quaestio de abortu“ (18. November 1974), AAS 66(1974): 730-47 (lat.), Zugriff am 16.12.2020, https://www.vatican.va/roman_curia/congregations/cfaith/documents/rc_con_cfaith _doc_19741118_declaration-abortion_ge.html (dt.). 
handelt werden dürfe (Nr. 9) beruft man sich sowohl auf „das göttliche Gesetz“ als auch auf „die natürliche Vernunft“, um den absoluten Ausschluss der Tötung von Unschuldigen zu rechtfertigen (Nr. 14). Gleichwohl bekennt die Glaubenskongregation die Existenz von echten Schwangerschaftskonflikten, in denen „die Verweigerung des Schwangerschaftsabbruches wichtige Güter verletzt, die man normalerweise schätzt und die selbst zuweilen vorrangig erscheinen können“ (Nr. 14), und expliziert diese Feststellung anhand einiger Beispiele, u. a. der Gefahr für Gesundheit und Leben der Mutter. Trotzdem heißt es anschließend: „Wir erklären nur, daß niemals einer dieser Gründe objektiv das Recht geben kann, über das Leben, selbst das beginnende, eines anderen zu verfügen" (Nr. 14).

Ein weiteres bioethisches Dokument der besagten Kongregation, Donum vitae von 1987, wird u. a. detaillierter im Hinblick auf den Beginn der unbedingten ethischen Schutzwürdigkeit des Menschen als Person, was traditionsentsprechend mit der Zeugung gegeben sein soll ${ }^{24}$.

Die Enzyklika Evangelium vitae von Johannes Paul II. (1995) ist gewissermaßen als Höhepunkt der lehramtlichen Verkündigung zur Abtreibungsfrage zu betrachten, nicht nur wegen des Ranges und der Ausführlichkeit des päpstlichen Dokumentes, sondern auch und vor allem, weil hier die Unfehlbarkeitskompetenz des Nachfolgers Petri eindeutig zur Geltung kommt, wie eingangs bereits erwähnt. Abgesehen von der absoluten Verurteilung der Abtreibung im Allgemeinen widmet sich der Papst auch dem Thema der lebensverkürzenden Erkrankungen, die Anlass für die sog. „eugenische“ Abtreibung geben, wobei er sich nicht scheut, mit scharfen Worten die dabei auftretenden Folgen als ethischen Regress anzuklagen ${ }^{25}$. Der Zusammenhang von dieser Form der Ab-

\footnotetext{
${ }^{24}$ Kongregation für die Glaubenslehre, Instruktion über die Achtung vor dem beginnenden menschlichen Leben und die Würde der Fortpflanzung „Donum vitae“ (22. Februar 1987), AAS 80(1988): 70-102 (lat.), Zugriff am 16.12.2020, https://www.vatican.va/roman_curia/congrega tions/cfaith/documents/rc_con_cfaith_doc_19870222_respect-for\%20human-life_ge.html (dt.): „Deshalb erfordert die Frucht der menschlichen Zeugung vom ersten Augenblick ihrer Existenz an, also von der Bildung der Zygote an, jene unbedingte Achtung, die man dem menschlichen Wesen in seiner leiblichen und geistigen Ganzheit sittlich schuldet. Ein menschliches Wesen muß vom Augenblick seiner Empfängnis an als Person geachtet und behandelt werden, und infolgedessen muß man ihm von diesem selben Augenblick an die Rechte der Person zuerkennen und darunter vor allem das unverletzliche Recht jedes unschuldigen menschlichen Wesens auf Leben“. (Teil I, 1).

25 „Die angebliche Rechtmäßigkeit der eugenischen Abtreibung entsteht in der öffentlichen Meinung aus einer Mentalität [...], die das Leben nur unter bestimmten Bedingungen annimmt und Begrenztheit, Behinderung und Krankheit ablehnt. Infolge eben dieser Logik ist man soweit gegangen, Kindern, die mit schweren Schäden oder Krankheiten geboren wurden, die ele-
} 
treibung, der Kindestötung und der Euthanasie über ein utilitaristisches „Normalitätskonzept", welches den Wert des Lebens relativiert, wird wiederum durch den Passus über die Gefahren der Pränataldiagnostik verdeutlicht ${ }^{26}$. Besondere Aufmerksamkeit im genannten Kontext verdient auch die Aussage über die absolute Gleichheit aller im Hinblick auf das Lebensrecht ${ }^{27}$.

Im Weltkatechismus von $1992^{28}$ sind hierzu v. a. die folgenden Abschnitte relevant: 2261 (Mord an Unschuldigen), 2270 (Achtung der Würde und Anerkennung von Personenrechten von Empfängnis an), 2271 (Einigkeit der Tradition über die moralische Verwerflichkeit der Abtreibung), 2274 (Sittlichkeit der Pränataldiagnostik) sowie 2275 (Eingriffe am menschlichen Embryo).

Unter den jüngsten lehramtlichen Dokumenten zu bioethischen Themen ragt v. a. die Neue Charta für die Mitarbeiter im Gesundheitswesen des Päpstlichen Rates für die Pastoral im Krankendienst von 2016 heraus ${ }^{29}$. Darin wird weiterhin eine begriffliche Eindeutigkeit appliziert, nach der jede direkte Vernichtung eines menschlichen Lebens zwischen Zeugung und Ge-

mentarsten üblichen Behandlungen und sogar die Ernährung zu verweigern. Noch bestürzender wird das moderne Szenarium darüber hinaus durch da und dort auftauchende Vorschläge, auf derselben Linie wie das Recht auf Abtreibung sogar die Kindestötung für rechtmäßig zu erklären: damit würde man in ein Stadium der Barbarei zurückfallen, das man für immer überwunden zu haben hoffte“. - Johannes Paul II., Enzyklika „Evangelium vitae“, Nr. 14, AAS 87(1995): 416

26 „Da jedoch die Behandlungsmöglichkeiten vor der Geburt heute noch recht begrenzt sind, kommt es nicht selten vor, daß diese Verfahren in den Dienst einer Eugenetik-Mentalität gestellt werden, die die selektive Abtreibung in Kauf nimmt, um die Geburt von Kindern zu verhindern, die von Mißbildungen und Krankheiten verschiedener Art betroffen sind. Eine solche Denkart ist niederträchtig und höchst verwerflich, weil sie sich anmaßt, den Wert eines menschlichen Lebens einzig und allein nach Maßstäben wie ,Normalität' und physisches Wohlbefinden zu beurteilen und auf diese Weise auch der Legitimation der Kindestötung und der Euthanasie den Weg bahnt“. - ebd., Nr. 63, AAS 87(1995): 473.

27 „Was das Recht auf Leben betrifft, ist jedes unschuldige menschliche Lebewesen allen anderen absolut gleich [...] Im Hinblick auf die sittliche Norm, die die direkte Tötung eines unschuldigen Menschen verbietet, ,gibt es für niemanden Privilegien oder Ausnahmen. Ob einer der Herr der Welt oder der Letzte, »Elendeste« auf Erden ist, macht keinen Unterschied: Vor den sittlichen Ansprüchen sind wir alle absolut gleich"“ - ebd., Nr. 57; inneres Zitat: Johannes Paul II., Enzyklika „Veritatis splendor" über einige grundlegende Fragen der kirchlichen Morallehre (6. August 1993), Nr. 96, AAS 85(1993): 1209 (lat.), Zugriff am 16.12.2020, https:// www.vatic an.va/content/john-paul-ii/de/encyclicals/documents/hf_jp-ii_enc_06081993_veritatissplendor.html (dt.), Hervorhebungen im Original.

${ }^{28}$ Dt. Version: Katechismus der katholischen Kirche. Neuübersetzung aufgrund der Editio typica latina. Mit CD-ROM (München: Oldenbourg u. a., korr. Nachdruck, 2005).

${ }^{29}$ Pontificio Consiglio per gli Operatori Sanitari, Nuova carta degli Operatori sanitari (Città del Vaticano: Libreria Editrice Vaticana, 2016). 
burt einen Abtreibungscharakter im vollen Sinne des Wortes trage (Nr. 28). Die moralische Zulässigkeit der Pränataldiagnostik wird traditionskonform von der Intention abhängig gemacht: Dienst am Leben der Mutter und des Kindes vs. eugenische Mentalität (Nr. 35). Letztere ist auch der Grund der dezidierten Verwerfung der Präimplantationsdiagnostik (Nr. 36). Das Tabu der Verfügung über das Leben eines anderen wird abschließend wiederholt: Trotz aller Gegenargumente und realer Schwierigkeiten „kennt die moralische Norm, die die direkte Beseitigung eines unschuldigen menschlichen Lebewesens verbietet, keine Ausnahme“ (Nr. 53).

\section{SCHLUSSWORT}

Dieser kurze Überblick stellt ein eindeutiges Bild der lehramtlichen Verkündigung zur Abtreibung vor Augen, und zwar in einem Bereich, der gleichzeitig die Grundlage für den Umgang mit lebensverkürzenden Krankheiten ausmacht. Den unerschütterlichen Leitgedanken bildet dabei die Feststellung: Eine Handlung, die sich als direkte, willentliche Tötung eines Ungeborenen qualifizieren lässt, ist absolut verwerflich und kann durch kein noch so hochstehendes Gut gerechtfertigt werden. Samaritanus bonus fügt sich in diese Tradition ein, indem es einen besonderen Blick auf das Umfeld des Kindes - besonders auf dessen Familie - wirft und dieses Umfeld ebenfalls zum Objekt der integralen Fürsorge erklärt. Der „Humus“ dieser Perspektive ist - wie in den sonstigen dargestellten Dokumenten auch - die personalistische Sicht des Menschen. Von daher kann man mithilfe der Anwendung des Personalismus auf bioethische Fragen die kirchliche Lehre im Bereich der lebensverkürzenden Erkrankungen synthetisieren und auf ihre Gründe überprüfen.

\section{LITERATUR}

Bębrowicz, Grzegorz H., Hg. Połoznictwo. Podręcznik dla położnych i pielęgniarek. Warszawa: PZWL, 2005.

Dangel, Tomasz. „Wady letalne u noworodków. Opieka paliatywna jako alternatywa wobec aborcji i uporczywej terapii“. Standardy Medyczne 31, Nr. 9 (2007): 108-10. Zugriff am 16.12.2020. https://hospicjum.waw.pl/pliki/Artykul/1095_wadyletalneunoworodkow-2007.pdf.

Dangel, Tomasz. „Wady letalne u płodów i noworodków. Opieka paliatywna jako alternatywa wobec eugenicznej aborcji, eugenicznego dzieciobójstwa i uporczywej terapii“. Zugriff am 16.12.2020. https://www.hospicjum.waw.pl/pliki/Artykul/1132_wadyletalneuplodowinowo rodkow.pdf. 
Furman, Filip. „Jakie skutki wywołuje wyrok Trybunału Konstytucyjnego w sprawie warunków dopuszczalności aborcji?““. Zugriff am 21.12.2020. https://ordoiuris.pl/sites/default/files/ inline-files/Stanowisko_CNSiB_1.pdf.

Heilige Kongregation für die Glaubenslehre. Erklärung über den Schwangerschaftsabbruch „Quaestio de abortu“ (18. November 1974). AAS 66(1974): 730-47 (lat.). Zugriff am 16.12.2020https://www.vatican.va/roman_curia/congregations/cfaith/documents/rc_con_cfa ith_doc_19741118_declaration-abortion_ge.html (dt.).

Jarzębińska, Aneta. „Od diagnozy do towarzyszenia w żałobie - wsparcie rodzin z dzieckim z wadą letalną“. Interdyscyplinarne Konteksty Pedagogiki Specjalnej 22(2018): 375-91. Zugriff am 16.12.2020. https://pressto.amu.edu.pl/index.php/ikps/article/view/21199/20455.

Johannes Paul II. Ap. Schreiben „Ad tuendam fidem“ durch das einige Normen in den CIC und in den CCEO eingefügt werden (18. Mai 1998). AAS 90(1998): 457-61. Zugriff: 16.12.2020. https://www.vatican.va/roman_curia/congregations/cfaith/documents/rc_con_cfaith_doc_1998_ professio-fidei_ge.html.

Johannes Paul II. Enzyklika „Evangelium vitae“ über den Wert und die Unantastbarkeit des menschlichen Lebens (25. März 1995). AAS 87(1995): 401-522. Zugriff: 16.12.2020. https:// www.vatican.va/content/john-paul-ii/de/encyclicals/documents/hf_jp-ii_enc_25031995_evan gelium-vitae.html.

Johannes Paul II. Enzyklika „Veritatis splendor“ über einige grundlegende Fragen der kirchlichen Morallehre (6. August 1993). AAS 85(1993): 1133-1228 (lat.). Zugriff am 16.12.2020. https://www.vatican.va/content/john-paul-ii/de/encyclicals/documents/hf_jp-ii_enc_06081993 _veritatis-splendor.html.

Jungbauer, Johannes und Rainer Krockauer, Hg. Wegbegleitung, Trost und Hoffnung. Interdisziplinäre Beiträge zum Umgang mit Sterben, Tod und Trauer. Opladen u. a.: Barbara Budrich, 2013.

Katechismus der katholischen Kirche. Neuübersetzung aufgrund der Editio typica latina. Mit CD-ROM. München: Oldenbourg u. a., korr. Nachdruck, 2005.

„Komunikat po ogłoszeniu wyroku w sprawie ,Planowanie rodziny, ochrona płodu ludzkiego i warunki dopuszczalności przerywania ciąży““. Trybunał Konstytucyjny. Zugriff am 16.12.2020. https://trybunal.gov.pl/postepowanie-i-orzeczenia/komunikaty-prasowe/komu nikaty-po/art/11299-planowanie-rodziny-ochrona-plodu-ludzkiego-i-warunki-dopuszczalnosci -przerywania-ciazy.

Kongregation für die Glaubenslehre. Instruktion über die Achtung vor dem beginnenden menschlichen Leben und die Würde der Fortpflanzung „Donum vitae“ (22. Februar 1987). AAS 80(1988): 70-102 (lat.). Zugriff am 16.12.2020. https://www.vatican.va/roman_curia/congre gations/cfaith/documents/rc_con_cfaith_doc_19870222_respect-for\%20human-life_ge.html.

Kongregation für die Glaubenslehre. Lehrmäßiger Kommentar zur Schlussformel der „Professio fidei“ (29. Juni 1998). AAS 90(1998): 544-51 (lat.). Zugriff am 16.12.2020. https://www. vatican.va/roman_curia/congregations/cfaith/documents/rc_con_cfaith_doc_1998_professio-fi dei_ge.html (dt.).

Kongregation für die Glaubenslehre. „Samaritanus bonus“. Schreiben über die Sorge an Personen in kritischen Phasen und in der Endphase des Lebens (14. Juli 2020). Città del Vaticano: Libreria Editrice Vaticana, 2020. Zugriff am 16.12.2020. https://www.vatican. va/roman_curia/congregations/cfaith/documents/rc_con_cfaith_doc_20200714_samaritanus-bo nus_ge.html.

„Number of abortion procedures performed in European countries in 2018“. Statista. Accessed December 16, 2020. https://www.statista.com/statistics/866423/abortion-rate-europe/.

Pius XI. Enzyklika „Casti connubi“ über die christliche Ehe im Hinblick auf die gegenwärtigen Lebensbedingungen und Bedürfnisse von Familie und Gesellschaft und auf die diesbe- 
züglich bestehenden Irrtümer und Mißbräuche (31.12.1930). AAS 22(1930): 539-92 (lat.). Zugriff am 16.12.2020. https://www.stjosef.at/dokumente/casti_connubii.htm (dt.).

Pius XII. Discorso alle partecipanti al Congresso della Unione Cattolica Italiana Ostetriche (29. Oktober 1951). AAS 43(1951): 835-54.

Pius XII. Discorso ai partecipanti al Convegno del „Fronte della Famiglia“ e della Federazione delle Associazioni delle Famiglie (27.11.1951). AAS 43(1951): 855-65.

Pontificio Consiglio per gli Operatori Sanitari. Nuova carta degli Operatori sanitari. Città del Vaticano: Libreria Editrice Vaticana, 2016.

Pyttlik, Andrea und Johannes Jungbauer. „Im Leben, im Sterben und darüber hinaus - Eine qualitative Studie zur Trauerbegleitung von Eltern lebensverkürzend erkrankter Kinder“. In Wegbegleitung, Trost und Hoffnung. Interdisziplinäre Beiträge zum Umgang mit Sterben, Tod und Trauer, hrsg. v. Johannes Jungbauer, Rainer Krockauer, 125-42. Opladen u. a.: Barbara Budrich, 2013.

Skrzypczak, Jana und Jakub Kornacki. „Ciąża o przebiego niepowikłanym“. In Położnictwo. Podręcznik dla położnych i pielęgniarek, hrsg. v. Grzegorz H. Bębrowicz, 72-4. Warszawa: PZWL, 2005.

„Ustawa z dnia 7 stycznia 1993 r. o planowaniu rodziny, ochronie płodu ludzkiego i warunkach dopuszczalności przerywania ciąży“. Internetowy System Aktów Prawnych. Zugriff am 01.02.2021. https://isap.sejm.gov.pl/isap.nsf/download.xsp/WDU19930170078/U/D199 30078Lj.pdf.

\author{
DIE ANTWORT DER KATHOLISCHEN KIRCHE AUF DIE FRAGE DER PERSONEN \\ MIT LEBENSVERKÜRZENDEN ERKRANKUNGEN IM LICHT DES SCHREIBENS \\ DER GLAUBENSKONGREGATION SAMARITANUS BONUS UND IM KONTEXT \\ DES URTEILS DES POLNISCHEN VERFASSUNGSGERICHTS VOM 22.10.2020
}

\title{
Z u s a m m e n a s s u n g
}

Das Urteil des polnischen Verfassungsgerichts vom 22.10.2020 bzgl. der Nichtkonformität der embryopatischen Indikation zur Abtreibung mit der Verfassung hat heftige Proteste ausgelöst. Im Zentrum der Auseinandersetzung befindet sich der Umgang mit lebensverkürzenden Erkrankungen, die für viele ein unannehmbares Übel und einen Grund für Abtreibung darstellen. Demgegenüber steht die Kirche seit jeher auf dem Boden des voraussetzungslosen Respekts vor dem Leben einer jeden menschlichen Person. Im folgenden Artikel geht es darum, im Ausgang vom polnischen Gerichtsurteil der Frage der Personen mit lebensverkürzenden Krankheiten ethisch nachzugehen, und zwar im Lichte eines neuen Dokuments der Glaubenskongregation Samaritanus bonus sowie in weiterer Perspektive der bisherigen kirchlichen Lehrverkündigung.

Schlagworte: lebensverkürzende Erkrankungen; Abtreibung; Samaritanus bonus; polnisches Verfassungsgericht; Person. 


\section{STANOWISKO KOŚCIOŁA KATOLICKIEGO WOBEC OSÓB Z WADAMI LETALNYMI W ŚWIETLE DOKUMENTU SAMARITANUS BONUS KONGREGACJI NAUKI WIARY W KONTEKŚCIE WYROKU POLSKIEGO TRYBUNAŁU KONSTYTUCYJNEGO Z 22 PAŹDZIERNIKA 2020 R.}

St re s z c z e n i e

Wyrok polskiego Trybunału Konstytucyjnego z dnia 22 października 2020 r. w sprawie niezgodności z konstytucją przesłanki o ciężkim i nieodwracalnym uszkodzeniu płodu jako wskazania do aborcji wywołał gwałtowne protesty. W centrum sporu znajduje się postępowanie w przypadku wad letalnych, które dla wielu są niedopuszczalnym złem i powodem do aborcji. W przeciwieństwie do tego Kościół od zawsze staje na gruncie bezwarunkowego szacunku dla życia każdego człowieka. Wychodząc od wspomnianego wyroku Trybunału, niniejszy artykuł dotyczy etycznego zbadania kwestii osób z chorobami letalnymi w świetle nowego dokumentu Kongregacji Nauki Wiary Samaritanus bonus, a także w szerszej perspektywie - dotychczasowego nauczania Kościoła.

Słowa kluczowe: wady letalne; aborcja; Samaritanus bonus; polski Trybunał Konstytucyjny; osoba. 